\title{
A importância dos ensaios de toxicidade para o desenvolvimento e o registro de
}

\section{fitoterápicos no Brasil}

\author{
The importance of toxicity tests for development and phytotherapy registration \\ La importancia de las pruebas de toxicidad para el desarrollo y registro de medicina herbaria en
}

Brasil

Recebido: 01/09/2021 | Revisado: 10/09/2021 | Aceito: 28/09/2021 | Publicado: 30/09/2021

\author{
Maisa Gomes da Silva \\ ORCID: https://orcid.org/0000-0002-8307-6021 \\ Universidade Federal do Piauí, Brasil \\ E-mail: gomesmaisa@outlook.com \\ Mariely Mendes Furtado \\ ORCID: https://orcid.org/0000-0001-8828-4236 \\ Universidade Federal do Piauí, Brasil \\ E-mail: marielymf@live.com \\ Altamiro Teixeira Osório \\ ORCID: https://orcid.org/0000-0002-5005-1020 \\ Universidade Federal do Piauí, Brasil \\ E-mail: biroteixeira@hotmail.com \\ Ilmara Cecília Pinheiro da Silva Morais \\ ORCID: https://orcid.org/0000-0001-5600-4041 \\ Universidade Federal do Piauí, Brasil \\ E-mail: Ilmara.cecilia@ hotmail.com \\ Maurício Pires Moura do Amaral \\ ORCID: https://orcid.org/0000-0003-3762-6712 \\ Universidade Federal do Piauí, Brasil \\ E-mail: maur.moura@ ufpi.edu.br \\ Angélica Gomes Coêlho \\ ORCID: https://orcid.org/0000-0002-4024-7652 \\ Universidade Federal do Piauí, Brasil \\ E-mail: angelicacoelho13@gmail.com \\ Daniel Dias Rufino Arcanjo \\ ORCID: https://orcid.org/0000-0001-7021-2744 \\ Universidade Federal do Piauí, Brasil \\ E-mail: daniel.arcanjo@ufpi.edu.br
}

\begin{abstract}
Resumo
Os estudos toxicológicos não-clínicos funcionam com um alicerce no processo de desenvolvimento de novos fármacos, visto que antecipam riscos e, portanto, reduzem a probabilidade de um novo fármaco interferir no metabolismo celular prejudicando a saúde do indivíduo além de não cumprir sua função farmacológica no organismo. Assim, considerando o potencial terapêutico das plantas medicinais, o presente trabalho tem como objetivo descrever a importância dos ensaios toxicológicos e os aspectos atuais concernentes ao registro de medicamentos fitoterápicos no Brasil. Para realização deste trabalho foram pesquisadas bases de dados científicas, tais como: Periódicos CAPES e Scielo, tendo o banco de dados sido complementado com artigos da legislação brasileira. Não houve restrição de busca quanto ao idioma ao ano de publicação dos materiais científicos, mas sim, quanto à relevância do seu conteúdo para o presente artigo. Dessa forma, observou-se que o papel regulador da ANVISA é fundamental para evitar que medicamentos ineficazes, tóxicos e de má qualidade alcancem o mercado e ocasionem problemas como intoxicações, interações com outros medicamentos, danos terapêuticos, ou até mesmo óbitos. Assim, por ser uma prática tradicional de saúde e já revelada em diversos estudos como de utilidade terapêutica por uma parcela significativa da população, torna-se interessante discutir a situação atual dos fitoterápicos e a seriedade que se deve ter no seu desenvolvimento principalmente diante dos ensaios toxicológicos não-clínicos uma vez que eles podem evitar problemas de saúde pública a curto e longo prazo.
\end{abstract}

Palavras-chave: Medicamentos fitoterápicos; Legislação; Toxicologia.

\begin{abstract}
Non-clinical toxicological studies work as a foundation in the process of developing new drugs, as they anticipate risks and, therefore, reduce the probability of a new drug interfering with cellular metabolism, harming the health of
\end{abstract}


the individual, in addition to not fulfilling its pharmacological function in the body. Thus, considering the therapeutic potential of medicinal plants, this paper aims to describe the importance of toxicological tests and current aspects concerning the registration of herbal medicines in Brazil. In order to carry out this work, scientific databases were searched, such as: Periódicos CAPES and Scielo, and the database was complemented with articles from Brazilian legislation. There was no search restriction regarding the language and year of publication of the scientific materials, but rather regarding the relevance of their content for this article. Thus, it was observed that the regulatory role of ANVISA is essential to prevent ineffective, toxic and poor quality drugs from reaching the market and causing problems such as intoxication, interactions with other drugs, therapeutic damage, or even death. Thus, as it is a traditional health practice and has already been revealed in several studies as being of therapeutic utility by a significant portion of the population, it is interesting to discuss the current situation of herbal medicines and the seriousness that should be taken in their development, especially in light of the trials non-clinical toxicologicals as they can prevent public health problems in the short and long term.

Keywords: Phytotherapeutic drugs; Legislation; Toxicology.

\section{Resumen}

Los estudios toxicológicos no clínicos funcionan como base en el proceso de desarrollo de nuevos fármacos, ya que anticipan riesgos y, por tanto, reducen la probabilidad de que un nuevo fármaco interfiera con el metabolismo celular, perjudicando la salud del individuo, además de no cumplir con su función. función farmacológica en el cuerpo. Así, considerando el potencial terapéutico de las plantas medicinales, este trabajo tiene como objetivo describir la importancia de las pruebas toxicológicas y los aspectos actuales relacionados con el registro de medicinas a base de hierbas en Brasil. Para la realización de este trabajo se realizaron búsquedas en bases de datos científicas, tales como: Periódicos CAPES y Scielo, y se complementó la base de datos con artículos de la legislación brasileña. No hubo restricción de búsqueda con respecto al idioma y año de publicación de los materiales científicos, sino más bien con respecto a la relevancia de su contenido para este artículo. Así, se observó que el rol regulador de ANVISA es fundamental para evitar que medicamentos ineficaces, tóxicos y de mala calidad lleguen al mercado y causen problemas como intoxicación, interacciones con otros medicamentos, daño terapéutico o incluso la muerte. Así, como es una práctica de salud tradicional y ya ha sido revelada en varios estudios como de utilidad terapéutica por una parte importante de la población, es interesante discutir la situación actual de las medicinas a base de hierbas y la seriedad que se debe tomar en su tratamiento. desarrollo, especialmente a la luz de los ensayos toxicológicos no clínicos ya que pueden prevenir problemas de salud pública a corto y largo plazo.

Palabras clave: Medicamentos fitoterápicos; Legislación; Toxicología.

\section{Introdução}

Caracterizada pelo uso de plantas medicinais em suas diferentes formas farmacêuticas, sem a utilização de substâncias ativas isoladas (Brasil, 2006), a fitoterapia é uma prática bastante antiga entre todas as civilizações e faz parte da cultura de muitos brasileiros (Feitosa et al., 2016). Sendo o Brasil o "país da diversidade", cujas culturas nativas foram o berço do conhecimento do qual hoje dispomos, é fundamental ressaltar a importância da medicina popular no desenvolvimento da indústria farmacêutica, visto que, a maioria dos medicamentos utilizados na atualidade foi originado de estudos desenvolvidos a partir da cultura popular, que fazem da rica biodiversidade brasileira um vasto campo de pesquisa científica (Lorenzi \& Matos, 2008).

Classificado como um produto de origem vegetal, utilizado como paliativo, profilático ou curativo, podendo ser simples ou composto a depender do número de espécies que foi extraído (Brasil, 2014), o desenvolvimentos dos fitoterápicos encara problemas referentes tanto à padronização dos fitopreparados para testes quanto aos aspectos normativos que são extremante variáveis entre os países, o que entrava a construção do conhecimento dos dados de segurança e eficácia dos novos fitoterápicos e dos já existentes (Lorenzi \& Matos, 2008).

Os estudos toxicológicos não-clínicos funcionam com um alicerce no processo de desenvolvimento de novos fármacos, visto que antecipam riscos e, portanto, reduzem os efeitos adversos e deletérios ao ser humano (Dorato \& Buckley, 2006). Esse tipo de estudo, pode ser realizado através de testes in vitro e in vivo. Os testes in vitro tem recebido bastante atenção em decorrência da coerção social para reduzir o número de animais usados na pesquisa e desenvolvimento de novos fármacos (Knop \& Maria, 2016). Todavia, são os testes in vivo que fornecem informações mais precisas sobre a segurança e eficácia dos novos fármacos (Bednarczuk et al., 2010). 
Sendo a toxicidade a principal causa de atrito de compostos em todos os estágios de descoberta e desenvolvimento de medicamentos, o número de interessados em demonstrar a importância dos ensaios de toxicidade no decorrer do processo de desenvolvimento de novos fármacos cresceu consideravelmente, cooperando cada vez mais para a compreensão da relação dose-resposta, assim como para seus efeitos sobre o ser humano (Dorato \& Buckley, 2006). Nessa perspectiva, um ensaio toxicológico, compreende uma análise completa de uma substância química com o intuito de produzir informações que assegurem ou não seu uso (Fonseca et al., 2008).

Para o registro de plantas medicinais na forma de derivados de drogas vegetais ou fitoterápicos e sua disponibilização à população, a Agência Nacional de Vigilância Sanitária (ANVISA) exige que o produto esteja criteriosamente avaliado principalmente quanto ao seu potencial toxicológico, objetivando ofertar um produto de qualidade e que não ofereça risco à saúde do homem (Brasil, 2014; Carvalho et al., 2007). Dessa forma, a Organização Mundial de Saúde (OMS) vem estimulando a inserção da fitoterapia nos sistemas de saúde de diversos os países. Assim, o interesse popular e institucional no Brasil vem crescendo no sentido de fortalecer a fitoterapia no Sistema Único de Saúde (SUS) (Brasil, 2006).

O progressivo crescimento dos fitoterápicos como alternativa aos medicamentos convencionais tem aumentado em todas as classes sociais das mais diversas regiões do mundo. Dados da OMS apontam que, em 1980, o mercado mundial de fitoterápicos e produtos naturais movimentou 500 milhões de dólares, o equivalente ao volume de vendas apenas da Europa no ano 2000 (Bruning et al., 2012). As preocupações com a biodiversidade, a busca por uma melhor qualidade de vida, o alto custo dos medicamentos industrializados (Lorenzi \& Matos, 2008) e o desenvolvimento de fitoterápicos reconhecidamente seguros e eficazes (Bruning et al., 2012) foram alguns dos fatores que alavancaram o crescimento do consumo de fitoterápicos pela população. Em vista disso, o presente artigo trata sobre a importância dos ensaios toxicológicos e os aspectos atuais concernentes ao registro de medicamentos fitoterápicos no Brasil.

\section{Metodologia}

A base metodológica utilizada para o trabalho foi a revisão bibliográfica narrativa descrita por Rother (2007) como sendo um método que permite o desenvolvimento de artigos nos quais os autores, analisam e interpretam de maneira mais abrangente e crítica os fenômenos sob uma ótica teórico ou contextual. O autor ainda chama atenção para a necessidade de ater-se a fontes de qualidade que assegurem uma análise profunda e fidedigna das informações, tendo sido o suporte metodológico embasado na obra Koche, J. C. (2011).

Para realização deste trabalho foram pesquisadas bases de dados científicas, tais como: Periódicos CAPES e Scielo, tendo o banco de dados sido complementado com artigos da legislação brasileira (Portarias, RDCs, RE, Decretos, Leis). Vale salientar que não houve restrição de busca quanto ao idioma ao ano de publicação dos materiais científicos, mas sim, quanto à relevância do seu conteúdo para o presente artigo. Foram utilizados basicamente autores que apresentassem informações consideráveis para uma análise consistente do referido contexto.

\section{Aspectos Regulatórios para o Registro e Desenvolvimento de um Fitoterápico}

O desenvolvimento de um medicamento fitoterápico compreende uma série de etapas, tais como: farmacobotânica, fitoquímica, farmacotécnica, e de estudos não clínicos e clínicos. Estes últimos abrangem estudos farmacológicos e toxicológicos a fim de garantir segurança e eficácia na viabilidade de produção de um fitoterápico (Oliveira et al., 2007). Entretanto, no Brasil a realização de pesquisas e a produção de medicamentos nesse ramo encaram problemas referentes à produção de insumos com qualidade necessária. Há limitações também no processo burocrático de acesso ao patrimônio genético, que desestimulam a pesquisa com biodiversidade. Além disso, há uma insegurança em relação às normas, que 
mudam frequentemente e não são consideradas com o mesmo rigor pelo conjunto de seus analistas, nos quais falta qualificação técnica. Dessa forma, a regulação é considerada um dos principais entraves do setor (Hasenclever et al., 2017).

$\mathrm{Na}$ tentativa de padronizar os critérios para avaliação dos fitoterápicos, a Organização Mundial de Saúde (OMS) reavaliou e escreveu novas monografias de plantas medicinais e editou o WHO Monographs on Selected Medicinal Plant volumes I e II, e o General Guidelines for methodologies on research and evaluation of traditional medicine, uma vez que grande parte da população dos países em desenvolvimento utiliza-se de práticas tradicionais na atenção primária a saúde, sendo boa parte destas o uso de plantas medicinais (Carvalho et al., 2007).

No Brasil, a Agência Nacional de Vigilância Sanitária (ANVISA) vem elaborando normas para a regulamentação destes medicamentos. Em 1967, com a portaria n ${ }^{\circ}$ 22, instituiu-se o Serviço Nacional de Fiscalização da Medicina e da Farmácia (SNFMF), implementando normas para o emprego de preparações fitoterápicas (Brasil, 1967) tornando necessários, para a sua produção, a realização de ensaios farmacológicos não-clínicos e clínicos. Em 1976, foi publicada a Lei no 6.360 , que dispõe sobre os produtos submetidos ao controle da Vigilância Sanitária (Brasil, 1976). Em 1995, a portaria no 6 estabeleceu prazos para que as indústrias farmacêuticas apresentassem dados de eficácia e segurança dos medicamentos fitoterápicos (Brasil, 1995). Quase uma década depois, em 2004, a RDC n ${ }^{\circ} .48$ dispõe sobre os registros de medicamentos fitoterápicos ${ }^{16}$. As RDCs no 267 e no 219 publicadas em 2005 e 2006, respectivamente, determinam que o cadastro de plantas destinadas à infusão extemporânea, para o preparo de chás, deve ser feito junto à Gerência de Alimentos da ANVISA (Anvisa, 2005; Anvisa, 2006).

Ainda em 2006, duas portarias relacionadas às plantas medicinais e fitoterápicos no Brasil foram publicadas. A primeira foi a portaria Ministerial $\mathrm{n}^{\circ}$ 971, de 03 de maio de 2006, que aprova a Política Nacional de Práticas Integrativas e Complementares (PNPIC) no SUS. A segunda foi o Decreto n ${ }^{\circ} 5.813$, que aprova a Política Nacional de Plantas Medicinais e Fitoterápicos (PNPMF) e dá outras providências. Ambas as políticas apresentaram em suas diretrizes o incentivo à pesquisa e desenvolvimento com relação ao uso de plantas medicinais e fitoterápicos e estimularam a adoção da fitoterapia nos programas de saúde pública (Brasil, 2006; Brasil, 2006).

Assim, em virtude de uma corrente naturalista que afetou o país anos depois, o consumo de plantas medicinais aumentou, resultando no surgimento de várias regulamentações complementares (Turolla \& Nascimento, 2006). Através da RDC $\mathrm{n}^{\circ}$ 49, de 23 de novembro de 2010, a ANVISA aprovou a Farmacopeia Brasileira 5. ${ }^{\text {a }}$ edição, elaborada com a participação de Instituições de Ensino e Pesquisa, revogando todas as monografias e métodos gerais das edições anteriores (Anvisa, 2010). Em 2012 por meio da Portaria GM/MS nº 533, a RENAME foi publicada abrangendo 12 fitoterápicos. Tal fato objetiva padronizar os medicamentos distribuídos no SUS e orientar ações no âmbito do complexo econômico-industrial da saúde (Brasil, 2012).

A regulamentação em vigor para o registro de medicamentos fitoterápicos é a RDC 26/2014, que determina os aspectos essenciais ao registro, como identificação botânica das espécies vegetais utilizadas, padrão de qualidade e identidade, provas de eficácia e segurança que validem as indicações terapêuticas propostas. Além do mais, estabelece as categorias de medicamento fitoterápico e produto tradicional fitoterápico e determina os requisitos mínimos para o registro e renovação de registro de medicamento fitoterápico, e para o registro, renovação de registro e notificação de produto tradicional fitoterápico (Brasil, 2014).

Nessa perspectiva, a distribuição e ampliação de fitoterápicos no SUS e o progressivo amadurecimento da legislação brasileira em relação aos estudos de eficácia e segurança destes medicamentos se antecipam ao aumento no número de medicamentos fitoterápicos a serem registrados (Carvalho et al., 2008). Sendo assim, a necessidade de estudos toxicológicos e a implantação de políticas de fitofarmacovigilância devem ser tomadas como prioridade para a saúde pública (Lanini et al., 2009). De forma resumida, a Figura 1 traz os principais marcos normativos envolvendo os fitoterápicos no Brasil. 
Figura 1: Linha do tempo dos principais aspectos normativos envolvidos no processo de elaboração, desenvolvimento, registro e fiscalização de produtos vegetais e seus derivados.

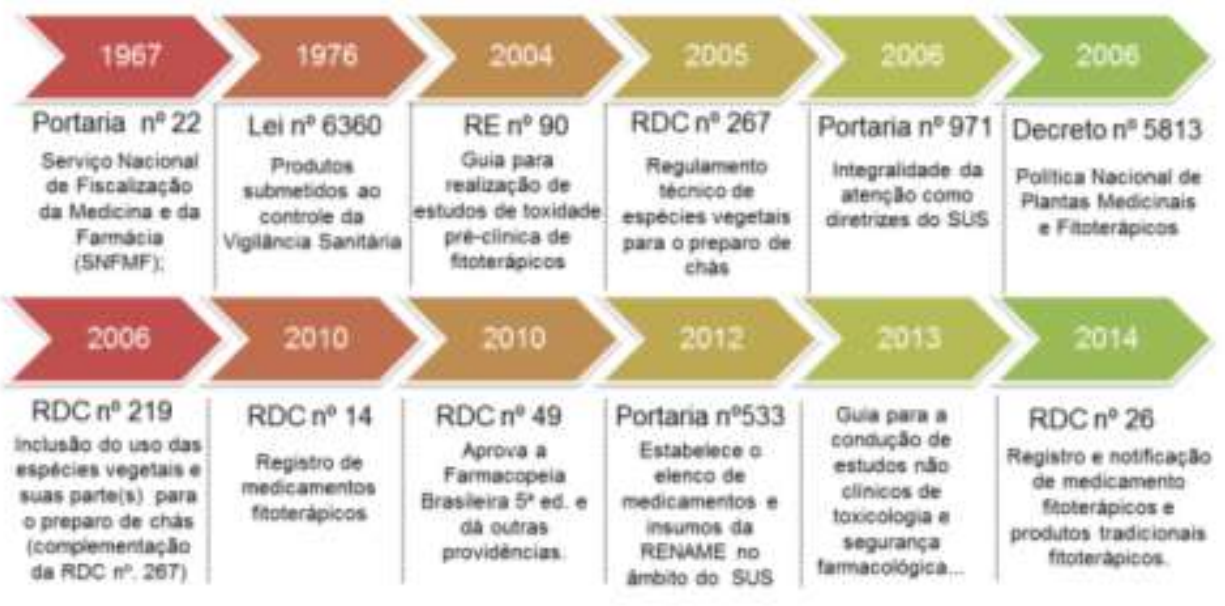

Fonte: Autores.

\section{Aspectos Normativos na Realização de Estudos Toxicológicos}

O primeiro guia para a realização de ensaios de toxicidade pré-clínica específico para medicamentos fitoterápicos só foi publicado em 2004, através da RE $n^{\circ}$ 90. Além dos ensaios de toxicidade aguda e de doses repetidas, essa resolução também preconiza estudos de genotoxicidade quando houver indicação de uso contínuo ou prolongado do medicamento em humanos e a avaliação toxicológica de fitoterápicos de uso tópico. Contudo, ela não descreve os métodos para a execução destes testes (Brasil, 2004).

Nessa perspectiva, a ANVISA divulgou, em 2010, a primeira versão do "Guia para a Condução de Estudos não Clínicos de Toxicologia e Segurança Farmacológica Necessários ao Desenvolvimento de Medicamentos”, objetivando maior conformidade com a regulamentação internacional, além da racionalização dos ensaios não clínicos. Evitando assim as duplicidades e o uso desnecessário de animais, sem interferir na obtenção e na confiabilidade dos resultados referentes à segurança da droga a ser testada, fornecendo dados confiáveis para as futuras pesquisas clínicas. Dessa maneira, é oportuno lembrar que o guia já está em sua segunda edição (Brasil, 2013).

Tais documentos foram elaborados em conformidade com as normas da Organização Mundial da Saúde (OMS) e da Organização para a Cooperação e Desenvolvimento Econômico (OECD). Dentre as recomendações internacionais feitas pela OECD para o Teste de Produtos Químicos, pode-se destacar o Guideline 423 (toxicidade aguda) e Guideline 407 (toxicidade subaguda) que apresentam procedimentos reproduzíveis, que usam poucos animais e são capazes de classificar substâncias de maneira semelhante a outros métodos de teste de toxicidade (Diretrizes de Teste 420 e 425). Esses e outros ensaios baseados nas diretrizes da OECD foram desenvolvidos por especialistas da área com o propósito de dar credibilidade aos resultados dos estudos toxicológicos permitindo comparar, reproduzir, avaliar e conferir uniformidade aos produtos avaliados tendo sido reconhecidos em atividades de pesquisa no Brasil pela Portaria Normativa $\mathrm{n}^{\circ} 18$ de 24 de setembro de 2014, do Conselho Nacional de Controle de Experimentação Animal - CONCEA (Brasil, 2014).

O estudo de toxicidade aguda é aquele aplicado para avaliar a toxicidade produzida por uma substância teste quando esta é administrada em uma ou mais doses durante um período não superior a 24 horas (Brasil, 2013). Com ele é possível evidenciar risco de intoxicações agudas, dando suporte à escolha das doses para os demais testes de toxicidade. Portanto, as informações obtidas por meio deste estudo são importantes na avaliação da segurança de substâncias químicas para uso humano e animal, entre as quais também se encontram as drogas vegetais e fitoterápicas (Madalosso, 2011). Por sua vez, os 
estudos de toxicidade subaguda têm como objetivo, caracterizar o perfil toxicológico da substância pela administração repetida. Baseado nele, é possível obter informações sobre os efeitos tóxicos, identificar órgãos alvos, alterações fisiológicas evidenciadas pelos parâmetros bioquímicos, hematológicos, anatômicos e histopatológicos. As doses empregadas nesses estudos geralmente são estipuladas a partir das informações concebidas em estudos de toxicidade aguda ou testes piloto para indicação de doses (Brasil, 2013).

A genotoxicidade avalia o que perturba a vida ou induz a morte tanto em nível de célula como de organismo. Testes genotóxicos podem ser realizados tanto in vitro quanto in vivo e são empregados para a detecção de substâncias que induzem dano genético, mutação genética, quebra cromossômica e alteração na capacidade de reparo de DNA (Brasil, 2013), sendo o teste cometa um dos ensaios de genotoxicidade mais aplicado devido ao baixo custo, alta sensibilidade e rapidez, servindo para a investigação e monitorização de dano ao material genético (Hartmann, 2003). Sob tal enfoque, a Tabela 1 resume as principais normas para a realização desses ensaios toxicológicos e as principais diferenças entre eles.

Tabela 1: Parâmetros preconizados para realização de estudos de toxicidade aguda, subagudo e genotoxicidade in vivo segundo o Guia para a Condução de estudos não clínicos de toxicologia e segurança farmacológica necessários ao desenvolvimento de medica.

\begin{tabular}{|c|c|c|c|}
\hline Parâmetros & Toxicidade Aguda & Toxicidade Subaguda & Genotoxicidade in vivo \\
\hline $\begin{array}{l}\text { Espécies de } \\
\text { animais }\end{array}$ & $\begin{array}{l}\text { Devem ser conduzidos com } \\
\text { no mínimo duas espécies de } \\
\text { mamíferos. }\end{array}$ & $\begin{array}{l}\text { Duas espécies de mamíferos, } \\
\text { incluindo uma espécie não } \\
\text { roedora ( } \mathrm{N}^{\mathrm{o}} \text { iguais de machos e } \\
\text { fêmeas) }\end{array}$ & $\begin{array}{ll}\text { Recomenda-se a utilização de } & \text { de } \\
\text { roedores (Camundongos } & \text { ou } \\
\text { ratos), de preferência machos; }\end{array}$ \\
\hline $\begin{array}{l}\text { Via de } \\
\text { Administração }\end{array}$ & $\begin{array}{l}\text { A pretendida para a } \\
\text { administração em humanos e } \\
\text { a parenteral; }\end{array}$ & $\begin{array}{l}\text { Devem ser utilizada a via em que } \\
\text { a droga será administrada em } \\
\text { humanos; }\end{array}$ & $\begin{array}{l}\text { Similares àquelas preconizadas } \\
\text { para uso humano; }\end{array}$ \\
\hline Dosagens & $\begin{array}{l}\text { A dose limite será } 1000 \\
\mathrm{mg} / \mathrm{kg} / \text { dia para roedores e não } \\
\text { roedores; }\end{array}$ & $\begin{array}{l}\text { Geralmente } 3 \text { doses; A mais alta } \\
\text { deve ser escolhida com a } \\
\text { expectativa de produzir efeitos } \\
\text { tóxicos observáveis respeitando- } \\
\text { se o limite máximo de } 1000 \\
\mathrm{mg} / \mathrm{kg} / \mathrm{dia} \text {; }\end{array}$ & $\begin{array}{l}\text { Três níveis de doses sendo que a } \\
\text { dose máxima não deve ultrapassar } \\
1000 \mathrm{mg} / \mathrm{kg} / \mathrm{dia} \text { para estudos de } \\
14 \text { dias ou mais; }\end{array}$ \\
\hline $\begin{array}{l}\text { Frequência de } \\
\text { administração }\end{array}$ & $\begin{array}{l}\text { Uma ou mais doses durante } \\
\text { um período não superior a } 24 \\
\mathrm{~h} \text {; }\end{array}$ & Uma dose diária & Uma dose diária \\
\hline $\begin{array}{l}\text { Período de } \\
\text { Observação }\end{array}$ & $\begin{array}{l}14 \text { dias após a administração } \\
\text { da substância teste. No dia da } \\
\text { administração os animais } \\
\text { devem ser observados no } \\
\text { mínimo duas vezes; }\end{array}$ & Mínimo de 2 semanas; & Mínimo de 2 semanas; \\
\hline
\end{tabular}

Fonte: Autores.

\section{Análise Toxicológica de Plantas Medicinais e Fitoterápicos}

Muitas substâncias químicas apresentam um potencial efeito tóxico, cujo efeito varia em função da concentração. Logo, a toxicidade é definida como a capacidade de uma substância, sob certas condições, de causar algum efeito danoso sobre o organismo, devido às suas propriedades químicas e à sua concentração (Fonseca et al., 2008). Dessa maneira, os ensaios toxicológicos podem ser subdividos em in vitro e in vivo, sendo este ainda classificado em agudo, subagudo, subcrônico e crônico, diferindo entre si principalmente sob o tempo de exposição do organismo-teste a substância e seu potencial de extensão tóxico (Knie \& Lopes, 2004). 
Em virtude da ascensão da ciência biomédica moderna no século XIX, registrou-se um aumento no número de animais utilizados em experiências. Assim, em 1959 com a publicação do livro "Principles of Human Experimental Technique" pelos pesquisadores William Russel e Rex Burch iniciou-se o movimento de proteção aos animais usados em experimentação ensino (Vicente \& Costa, 2014). Tal acontecimento representou um marco na discussão sobre a utilização de animais para a avaliação de toxicidade implantando assim, o princípio dos 3R's (Reduction, Refinement e Replacement) (Cazarin et al., 2004). A redução reflete a obtenção de nível equiparável de informação com o uso de menos animais; o refinamento promove o alívio ou a minimização da dor, sofrimento ou estresse do animal; a substituição estabelece que um determinado objetivo seja alcançado sem o uso de animais vertebrados vivos. Dessa maneira, os métodos alternativos podem ser definidos como qualquer método que possa ser usado para substituir, reduzir ou refinar o uso de animais de experimentação na pesquisa biomédica, ensaios ou ensino (Vicente \& Costa, 2014).

Ensaios de toxicidade in vitro estão sendo amplamente difundido, uma vez que eles anteveem a toxicidade de um ativo em seres humanos mediante a utilização de micro-organismos, enzimas e culturas celulares (Bednarczuk et al., 2010), sendo a técnica de cultivo celular o método in vitro mais empregado no estudo toxicológico, posto que o desenvolvimento de novos fármacos surge a partir destes. Além do mais, a execução dos testes in vitro é mais simples e mais rápido que a do teste in vivo e podem substituir os animais ou, ao menos, servirem como uma análise preliminar aos testes in vivo (Rogero et al., 2003).

Nessa perspectiva, os ensaios in vitro demonstram-se mais vantajosos em relação aos testes in vivo, uma vez que propiciam um maior controle do meio, boa reprodutividade dos ensaios, simplicidade, rapidez e baixo custo atenuando o número de animais utilizados nos experimentos. Outra grande vantagem é a diversidade de linhagens celulares disponíveis no mercado, fazendo com que os testes de citotoxicidade in vitro já sejam apontados como substitutos dos testes de toxicidade aguda, dado que a indústria farmacêutica necessita de novas técnicas e modelos alternativos para determinação do perfil toxicológico, a fim de melhorar a pesquisa (Benauer et al., 2005).

Apesar dos avanços nestes ensaios, a extrapolação direta dos pontos finais do ensaio in vitro para os efeitos adversos relevantes para os sistemas in vivo é limitada uma vez que não temos disponíveis linhagens celulares adequadas que imite apropriadamente a resposta in vivo e os processos de ativação e inativação metabólica não são preservados integralmente nos ensaios in vitro (Lauterstein et al., 2020). Dessa maneira, os resultados adquiridos com testes in vitro ajudam a delinear os ensaios que deverão ser realizados in vivo além de direcionar quais substâncias serão testadas em tais ensaios (Benauer et al., 2005).

Nessa perspectiva, o grau de segurança no uso de drogas vegetais só pode ser avaliado quando se analisa uma série de fatores, tais como as propriedades físicas, químicas e farmacodinâmicas das drogas, além das peculiaridades biológicas dos indivíduos, pois durante os testes de toxicidade in vivo, os animais ou humanos usados nos estudos devem ser avaliados periodicamente quanto às alterações físicas e comportamentais, sendo a interpretação dos resultados específica para cada constituinte, apesar de existirem princípios básicos e associações que podem ser seguidas (Kerr, 2003).

O catastrófico caso da Talidomida, na década de 1960 apontou deficiências no emprego dos roedores em prever riscos de toxicidade em humanos, demonstrando o quão versátil pode ser a resposta aos fármacos. Todavia, apesar das particularidades existentes entre as espécies, no que diz respeito à absorção, distribuição, biotransformação e eliminação, e da variabilidade de resposta, o feedback de um modelo vivo é mais confiável do que qualquer outro modelo (Pinto et al., 2010).

Nesse sentido, o ensaio de toxicidade aguda é um estágio primordial para a avaliação do perfil toxicológico de uma substância, podendo evidenciar o mecanismo de toxicidade envolvido na absorção da mesma por diferentes vias de administração. Este ensaio pode ser relevante para a determinação das doses a serem utilizadas em estudos subagudos e 
crônicos. Além disso, viabiliza informações sobre o risco à saúde humana advindo da administração aguda dessa substância (Bighetti et al., 2004).

A propósito da toxicidade latente em todas as substâncias, a determinação da dose letal média (DL50), definida como a quantidade em miligramas por quilograma de peso corporal necessária para provoca a morte de $50 \%$ de um grupo de animais da mesma espécie, quando administrada pela mesma via (Minho et al., 2016), não é mais requerida pela FDA desde 1983 nos processos regulatórios em virtude da implantação de métodos alternativos eficazes e menos agressivo aos animais (Cazarin $e t$ al., 2004).

Desse modo, os primeiros guias de procedimentos alternativos validados e adotados pela OECD demonstraram que avaliações toxicológicas agudas utilizando um número menor de animais do que o teste clássico de DL50 também podem fornecer respostas adequadas de toxicidade aguda e letalidade. Embora a letalidade ainda seja o foco principal, estes ensaios reduzem o número e o sofrimento dos animais sem deixar de fornecer informações adequadas para a classificação toxicológica das substâncias analisadas (Cazarin et al., 2004). Assim, em 2001, estas normas foram atualizadas e o Guia OECD No 401 referente à toxicidade aguda e determinação da DL50 foi suspenso em diversos países (Botham, 2002). Em vista disso, cabe ao pesquisador selecionar o protocolo mais adequado a sua pesquisa uma vez que existem vários procedimentos sugeridos para desenvolvimento dos estudos de toxicidade de fármacos e atualmente, no Brasil, existe protocolo especifico para os fitoterápicos.

\section{Considerações Finais}

O papel regulador da ANVISA é fundamental para evitar que medicamentos ineficazes, tóxicos e de má qualidade alcancem o mercado e ocasionem problemas como intoxicações, interações com outros medicamentos, danos terapêuticos, ou até mesmo óbito. Assim, por ser uma prática tradicional de saúde e já revelada em diversos estudos como de utilidade terapêutica por uma parcela significativa da população, torna-se interessante discutir a situação atual dos fitoterápicos e a seriedade que se deve ter no seu desenvolvimento principalmente diante dos ensaios toxicológicos não-clínicos uma vez que eles podem evitar problemas de saúde pública a curto e longo prazo.

Diante do exposto, este trabalho de revisão elenca metodologias para avaliação de toxicidade de fitoterápicos e fornece subsídio a esta investigação em trabalhos futuros, sugerindo-se especialmente a adoção de metodologias alternativas validadas aceitas internacionalmente, de modo a racionalizar o uso de animais em estudos in vivo e gerar resultados confiáveis e reprodutíveis.

\section{Referências}

ANVISA- Agência Nacional de Vigilância Sanitária. RDC no 48, de 16 de março de 2004. Dispõe sobre o registro de medicamento fitoterápico. D.O.U. Diário Oficial União; Poder Executivo, 18 mar 2004. https://www.cpqba.unicamp.br/plmed/docs/Resolucao\%20RDC\%2048\%20de\%2016032004.PDF

ANVISA - Agência Nacional de Vigilância Sanitária. RDC nº 267, de 22 de setembro de 2005. Aprova o Regulamento Técnico de Espécies vegetais para o preparo de chás. D.O.U. - Diário Oficial da União; Poder Executivo, 23 de setembro de 2005. https://www.saude.rj.gov.br/comum/code/MostrarArquivo.php?C=MjIwNQ\%2C\%2C

ANVISA - Agência Nacional de Vigilância Sanitária. RDC nº 219, de 22 de dezembro de 2006. Aprova a inclusão do uso das espécies vegetais e parte(s) de espécies vegetais para o preparo de chás constante da Tabela 1 do Anexo desta Resolução em complementação as espécies aprovadas pela Resolução ANVISA RDC n. 267, de 22 de setembro de 2005. D.O.U. - Diário Oficial da União; Poder Executivo, de 26 de dezembro de 2006. https://bvsms.saude.gov.br/bvs/saudelegis/anvisa/2006/res0219_22_12_2006.html

ANVISA - Agência Nacional de Vigilância Sanitária. RDC N. ${ }^{\circ}$ 49, de 23 de novembro de 2010, Aprova a Farmacopeia Brasileira, $5^{\text {a }}$ edição e dá outras providências. Diário Oficial da União, Brasília-DF, 24 de novembro de 2010. https://www.diariodasleis.com.br/busca/exibelink.php?numlink=215633

Bednarczuk, V. O., Verdam, M. C. S., Miguel, M. D. \& Miguel, O. G. (2010). Testes in vitro e in vivo utilizados na triagem toxicológica de produtos naturais. Visão Acadêmica, 11(2), 43-50.

Bernauer, U., Oberemm, A., Madle, S. \& Gundert-Remy, U. (2005). The use of in vitro data in risk assessment. Basic \& clinical pharmacology \& toxicology, 96(3), 176-181. https://doi.org/10.1111/j.1742-7843.2005.pto960306.x 
Bighetti, A. E., Antônio, M. A., Possenti, A., Foglio, M. A., Siqueira, M. G. \& Carvalho, J. E. (2004). Efeitos da administração aguda e subcrônica da Luehea divaricata Martus et Zuccarini. Lecta-USF, 22(1/2), 53-58.

Brasil. Serviço de Fiscalização de Medicina e Farmácia. Portaria nº 22, de 30 de outubro de 1967. Estabelece normas para o emprego de preparações fitoterápicas. Diário Oficial da União. 16 nov 1967.

Brasil. Ministério da Saúde. Lei ${ }^{\circ}$ 6360, de 23 de setembro de 1976. Dispõe sobre a vigilância sanitária a que ficam sujeitos os medicamentos, as drogas, os insumos farmacêuticos e correlatos, cosméticos, saneantes e outros produtos, e dá outras Providências. Diário Oficial [da] República Federativa do Brasil, Brasília, DF, 24 setembro 1976. https://www.planalto.gov.br/ccivil_03/leis/16360.htm

Brasil. Portaria $\mathrm{n}^{\circ}$ 6, de 31 de janeiro de 1995. Instituir e normatizar o registro de produtos fitoterápicos junto ao Sistema de Vigilância Sanitária. Diário Oficial União. 6 fev 1995; Seção 1:1523.

Brasil. Agência Nacional de Vigilância Sanitária - (ANVISA). RE no 90, de 16 de março de 2004. Dispõe sobre o Guia para a realização de estudos de toxicidade pré-clínica de fitoterápicos. D.O.U.- Diário Oficial da União, Brasília-DF, 18 de março de 2004. https://www.diariodasleis.com.br/busca/exibelink.php?numlink=1-9-34-2004-03-16-90

Brasil. Ministério da Saúde. Portaria nº 971, de 3 de maio de 2006. Aprova a Política Nacional de Práticas Integrativas e Complementares (PNPIC) no Sistema

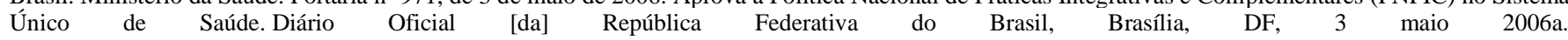
https://bvsms.saude.gov.br/bvs/saudelegis/gm/2006/prt0971_03_05_2006.html

Brasil. Decreto n 5813 de 22 de junho de 2006. Aprova a Política de Plantas Medicinais e Fitoterápicos e dá outras providências. D.O.U. - Diário Oficial da União; Poder Executivo, 22 de junho de 2006. http://www.planalto.gov.br/ccivil_03/_ato2004-2006/2006/decreto/d5813.htm

Brasil. Ministério da Saúde. Portaria no ${ }^{\circ}$ 533, de 28 de março de 2012. Estabelece o elenco de medicamentos e insumos da relação nacional de medicamentos essenciais (RENAME) no âmbito do Sistema Único de Saúde (SUS). D. O. U.- Diário Oficial da União, Brasília-DF, março de 2012. https://bvsms.saude.gov.br/bvs/saudelegis/gm/2012/prt0533_28_03_2012.html

Brasil. Agência Nacional de Vigilância Sanitária (ANVISA) - Guia para a Condução de Estudos Não clínicos de Segurança Necessários ao Desenvolvimento de Medicamentos. Brasília-DF, 2013. 48p. https://www.gov.br/anvisa/pt-br/centraisdeconteudo/publicacoes/medicamentos/pesquisa-clinica/manuais-eguias/guia-para-a-conducao-de-estudos-nao-clinicos-de-toxicologia-e-seguranca-farmacologica-necessarios-ao-desenvolvimento-de-medicamentos-versao2.pdf/view

Brasil. Ministério da Ciência, Tecnologia e Inovação. Resolução Normativa No 18, de 24 de setembro de 2014. Reconhece métodos alternativos ao uso de animais em atividades de pesquisa no Brasil, nos termos da Resolução Normativa ${ }^{\circ}$ 17, de 03 de julho de 2014, e dá outras providências. D.O.U. Seção I, Pág. 9, Brasilia-DF, 25 de setembro de 2014. https://portal.anhembi.br/wp-content/uploads/2019/10/Resolu\%C3\%A7\%C3\%A3o-Normativa-N\%C2\%BA-18de-24-de-setembro-de-2014.pdf

Brasil. Ministério da Saúde. RDC N 26, de 13 de maio de 2014. Dispõe sobre o registro de medicamentos fitoterápicos e o registro e a notificação de produtos tradicionais fitoterápicos. Diário Oficial [da] República Federativa do Brasil, Brasília, DF, 13 maio 2014. https://bvsms.saude.gov.br/bvs/saudelegis/anvisa/2014/rdc0026_13_05_2014.pdf

Bruning, M. C. R.; Mosegui, G. B. G.; Vianna, C. M. M. (2012). A utilização da fitoterapia e de plantas medicinais em unidades básicas de saúde nos municípios de Cascavel e Foz do Iguaçu - Paraná: a visão dos profissionais de saúde. Ciência \& Saúde Coletiva, 17(10), 2675-85.

Botham, P. A. (2002). Acute Systemic Toxicity. ILAR Journal, 43(Suppl_1), S27-S30. https://doi.org/10.1093/ilar.43.suppl_1.s27

Carvalho, A. C. B., Nunes, D. S. G., Baratelli, T.G. \& Shuqairnsmsaq, Netto, E. M. (2007). Aspectos da legislação no controle dos medicamentos fitoterápicos. $T \& C$ Amazônia, 11, 26-32.

Carvalho, A. C. B., Balbino, E. E., Maciel, A. \& Perfeito, J. P. S. (2008). Situação do registro de medicamentos fitoterápicos no Brasil. Revista Brasileira de Farmacognosia, 18(2), 314-319. https://doi.org/10.1590/s0102-695x2008000200028

Cazarin, K. C. C., Corrêa, C. L. \& Zambrone, F. A. D. (2004). Redução, refinamento e substituição do uso de animais em estudos toxicológicos: uma abordagem atual. Revista Brasileira de Ciências Farmacêuticas, 40(3), 289-299. https://doi.org/10.1590/s1516-93322004000300004

Dorato, M. A. \& Buckley, L. A. (2006). Toxicology in the Drug Discovery and Development Process. Current Protocols in Pharmacology. 32:10.3.1-10.3.35.

Feitosa, M. H. A., Soares, L. L., Borges, G. A., Andrade, M. M. \& Costa, S.M. (2016). Inserção do Conteúdo Fitoterapia em Cursos da Área de Saúde. Revista Brasileira de Educação Médica, 40(2),197-203.

Fonseca, J. C. L., Marchi, M. R. R. \& Fonseca, J. C. L. (2008). Programa Internacional de Segurança Química - Substâncias Químicas Perigosas à Saúde e ao Ambiente. São Paulo: Cultura Acadêmica, São Paulo-SP. p. 119. file:///C:/Users/marqu/Downloads/subs_quimicas\%20(3).pdf

Hartmann, A. (2003). Recommendations for conducting the in vivo alkaline Comet assay. Mutagenesis, 18(1), 45-51. https://doi.org/10.1093/mutage/18.1.45

Hasenclever, L., Paralhos, J., Costa, C. R., Cunha, G. \& Vieira, D. (2017). A indústria de fitoterápicos brasileira: desafios e oportunidades. Ciência \& Saúde Coletiva, 22(8), 2559-69.

Kerr, M. G. (2003). Exames laboratoriais em Medicina Veterinária: Bioquímica Clínica e Hematologia. 2 ed., parte II, pp 81-163. São Paulo: Roca.

Knie, J. L.W. \& Lopes, E.W. B. (2004). Testes ecotoxicológicos: métodos, técnicas e aplicações. Florianópolis: FATMA, 2004.

Knop, L. B. \& Maria, D. A. (2016). Métodos Substitutivos e a Experimentação Animal: um Enfoque Inovador. Revista da Sociedade Brasileira de Ciência em Animais de Laboratório, 4(2), 101-14. 
Research, Society and Development, v. 10, n. 12, e538101220137, 2021

(CC BY 4.0) | ISSN 2525-3409 | DOI: http://dx.doi.org/10.33448/rsd-v10i12.20137

Köche, J. C. (2011). Fundamentos de metodologia científica : teoria da ciência e iniciação à pesquisa. Petrópolis, RJ : Vozes, p. 185. http://www.adm.ufrpe.br/sites/ww4.deinfo.ufrpe.br/files/Fundamentos_de_Metodologia_Cienti\%CC\%81fica.pdf

Lanini, J., Duarte-Almeida, J. M., Nappo, S. \& Carlini, E. A. (2009). "O que vêm da terra não faz mal": relatos de problemas relacionados ao uso de plantas medicinais por raizeiros de Diadema/SP. Revista Brasileira de Farmacognosia, 19(1a), 121-129. https://doi.org/10.1590/s0102-695x2009000100022

Lauterstein, D., Savidge, M., Chen, Y., Weil, R. \& Yeager, R. P. (2020). Nonanimal toxicology testing approaches for traditional and deemed tobacco products in a complex regulatory environment: Limitations, possibilities, and future directions. Toxicology in Vitro, 62, 104684. https://doi.org/10.1016/j.tiv.2019.104684.

Lorenzi, H. \& Matos, F. J. de A. (2008). Plantas medicinais no Brasil: nativas e exóticas. 2. ed. Nova Odessa: Instituto Plantarum.

Madalosso, R. C. (2011) Avaliação da toxicidade aguda e da atividade gastroprotetora de extratos de Campomanesia lineatifolia Ruiz \& Pav. em roedores. Tese (Mestrado) Universidade Federal de Minas Gerais. Belo Horizonte, p. 1-116.

Minho, A. P., Gaspar, E. B. \& Domingues, R. Guia Prático para Determinação de Curva Dose-Resposta e Concentração Letal em Bioensaios com Extratos Vegetais, Comunicado Técnico - EMBRAPA, Bagé-RS, Abril. 2016. https://ainfo.cnptia.embrapa.br/digital/bitstream/item/172038/1/Comunicado-Tecnico-n93.pdf

Oliveira, A. B., Longhi, J. G., Andrade, C. A., Miguel, O. G. \& Miguel, M. D. A. (2007). Normatização dos Fitoterápicos no Brasil. Visão Acadêmica. 7(2).

Pinto, T. J. A., Kaneko, T. M.\& Pinto, A. F. (2010). Controle biológico de qualidade de produtos farmacêuticos, correlatos e cosmético (Cap. X, pp. 697772). São Paulo: Atheneu Editora.

Rogero, S. O., Lugão, A. B., Ikeda, T. I. \& Cruz, U. S. (2003). Teste in vitro de citotoxicidade: estudo comparativo entre duas metodologias. Materials Research, 6(3), 317-320. https://doi.org/10.1590/s1516-14392003000300003

Rother, E. T. (2007). Revisão sistemática x revisão narrativa. Acta Paul Enferm. 20(2),v-vi. https://doi.org/10.1590/S0103-21002007000200001

Turolla, M. S. R. \& Nascimento, E. S. (2006). Informações toxicológicas de alguns fitoterápicos utilizados no Brasil. Revista Brasileira de Ciências Farmacêuticas. 42(2).

Vicente, A. M. \& Costa, M. C. D. (2014). Experimentação animal e seus limites: core set e participação pública. Physis: Revista de Saúde Coletiva, 24(3), 831-849. https://doi.org/10.1590/s0103-73312014000300009 